\title{
Bioassay-guided isolation of antifungal plumericin from Allamanda species (Apocynaceae).
}

\begin{abstract}
Anthracnose is an economically important disease that can cause 10 to $80 \%$ yield reduction in the market with its typical symptoms of dark spots, sunken necrotic tissues and concentric rings of acervuli on fruits. One of the important methods to control the disease is by using antifungal agents derived from plant. Preliminary study indicated that the extracts of Allamanda species have the potential to be developed as the target agent. Five Allamanda species were extracted and screened for antifungal activity against plant pathogenic fungus Colletotrichum gloeosporioides by using poison agar technique. The three chloroform extracts of A. blanchetti, A. cathartica 'Alba' and A. cathartica 'Jamaican Sunset' exhibited potent inhibitory effects and suppressed the mycelial growth of C. gloeosporioides by up to about $70 \%$. Further study by bioautography-guided fractionation of the extracts led to the isolation and identification of tetracyclic sesquiterpene plumericin as the antifungal agent. The structural determination of the compound was carried out by interpreting the IR, MS, 1D-NMR and 2D-NMR spectral data.
\end{abstract}

Keyword: Allamanda species; Antifungal; Colletotrichum gloeosporioides; Bioautography; Plumericin. 